\title{
Diode quenching for Geiger mode avalanche photodiode
}

\author{
Jong-Ik Kang ${ }^{1}$, Hyuk-Kee Sung ${ }^{1}$, Hyungtak Kim ${ }^{1}$, \\ Eugene Chong ${ }^{2}$, and Ho-Young Cha ${ }^{1 \mathrm{a})}$ \\ ${ }^{1}$ School of Electronic and Electrical Engineering, Hongik University, \\ Seoul 04066, Korea \\ ${ }^{2}$ Chem-Bio Division, Agency for Defense Development, Daejeon 34186, Korea \\ a) hcha@hongik.ac.kr
}

Abstract: A resistive quenching element is a common passive device used in Geiger mode avalanche photodiode circuits. Both quenching voltage level and response time depend on the resistance value of the quenching resistor. A high resistance value of the quenching resistor increases the transient time due to the large RC time constant. In addition, the quenching voltage level depends on the photocurrent passing through the quenching resistor. In this work, a diode quenching element was proposed by which faster quenching speed was achieved with a constant quenching voltage level independent of the photocurrent level.

Keywords: APD, Geiger mode, UV detection

Classification: Optical systems

\section{References}

[1] J. C. Campbell, et al.: "Recent advances in avalanche photodiodes," IEEE J. Sel. Top. Quantum Electron. 10 (2004) 777 (DOI: 10.1109/JSTQE.2004. 833971).

[2] F. Yan, et al: " $4 \mathrm{H}-\mathrm{SiC}$ visible blind UV avalanche photodiode," Electron. Lett. 35 (1999) 929 (DOI: 10.1049/el:19990641).

[3] H. Y. Cha and P. M. Sandvik: "Electrical and optical modeling of 4H-SiC avalanche photodiodes,” Jpn. J. Appl. Phys. 47 (2008) 5423 (DOI: 10.1143/ JJAP.47.5423).

[4] H. Y. Cha: "Structural optimization of silicon carbide PIN avalanche photodiodes for UV detection,” J. Korean Phys. Soc. 56 (2010) 672 (DOI: $10.3938 / \mathrm{jkps.56.672).}$

[5] A. Vert, et al:: "Solar-blind 4H-SiC single-photon avalanche diode operating in Geiger mode," IEEE Photonics Technol. Lett. 20 (2008) 1587 (DOI: 10.1109/ LPT.2008.928852).

[6] H.-Y. Cha, et al.: "4H-SiC avalanche photodiodes for $280 \mathrm{~nm}$ UV detection," IEICE Trans. Electron. E93.C (2010) 648 (DOI: 10.1587/transele.E93.C.648).

[7] D. Zhou, et al:: "High-temperature single photon detection performance of 4H-SiC avalanche photodiodes," IEEE Photonics Technol. Lett. 26 (2014) 1136 (DOI: 10.1109/LPT.2014.2316793).

[8] A. Gallivanoni, et al:: "Progress in quenching circuits for single photon avalanche diodes," IEEE Trans. Nucl. Sci. 57 (2010) 3815 (DOI: 10.1109/TNS. 2010.2074213).

[9] T. Kagawa: "Simulation of the Geiger mode operation of a single photon detection avalanche photodiode," IEICE Trans. Electron. E86.C (2003) 1366. 
[10] V. Savuskan, et al.: "Selecting single photon avalanche diode (SPAD) passivequenching resistance: An approach," IEEE Sensors J. 13 (2013) 2322 (DOI: 10.1109/JSEN.2013.2253603).

[11] A. Dalla Mora, et al.: "Single-photon avalanche diode model for circuit simulations," IEEE Photonics Technol. Lett. 19 (2007) 1922 (DOI: 10.1109/ LPT.2007.908768).

[12] M. Liu, et al.: "Reduce afterpulsing of single photon avalanche diodes using passive quenching with active reset," IEEE J. Quantum Electron. 44 (2008) 430 (DOI: 10.1109/JQE.2007.916688).

\section{Introduction}

Avalanche photodiodes (APDs) are used to detect weak optical signals and have several advantages compared to photomultiplier tubes, such as, small size, low voltage operation, high quantum efficiency, insensitivity to the magnetic field, etc $[1,2,3]$. When an APD is biased above the breakdown voltage, the high electric field in the depletion region causes the self-sustained avalanche process by which the photo-generated current is multiplied with a high gain $[3,4]$.

A quenching circuit is used for triggering the APD bias voltage in the Geiger mode to the linear mode $[5,6,7]$. A resistive quenching circuit shown in Fig. 1 is a common topology in Geiger mode operation. The generated photocurrent causes a voltage drop across the quenching resistor $\left(\mathrm{R}_{\mathrm{q}}\right)$, which, in turn, lowers the bias voltage of the APD below the avalanche bias voltage. Since the quenching voltage level required in the circuit is typically a few volts, the resistance value of $R_{q}$ must be selected by taking account of the photocurrent level $[5,6,7,8]$. Typical resistance values of $R_{q}$ range from hundreds of $k \Omega$ to $M \Omega$ due to the low photocurrent levels. Such high resistance values lead to a large RC time constant that increases the dead time and thus limits the sensing capability. Employing an

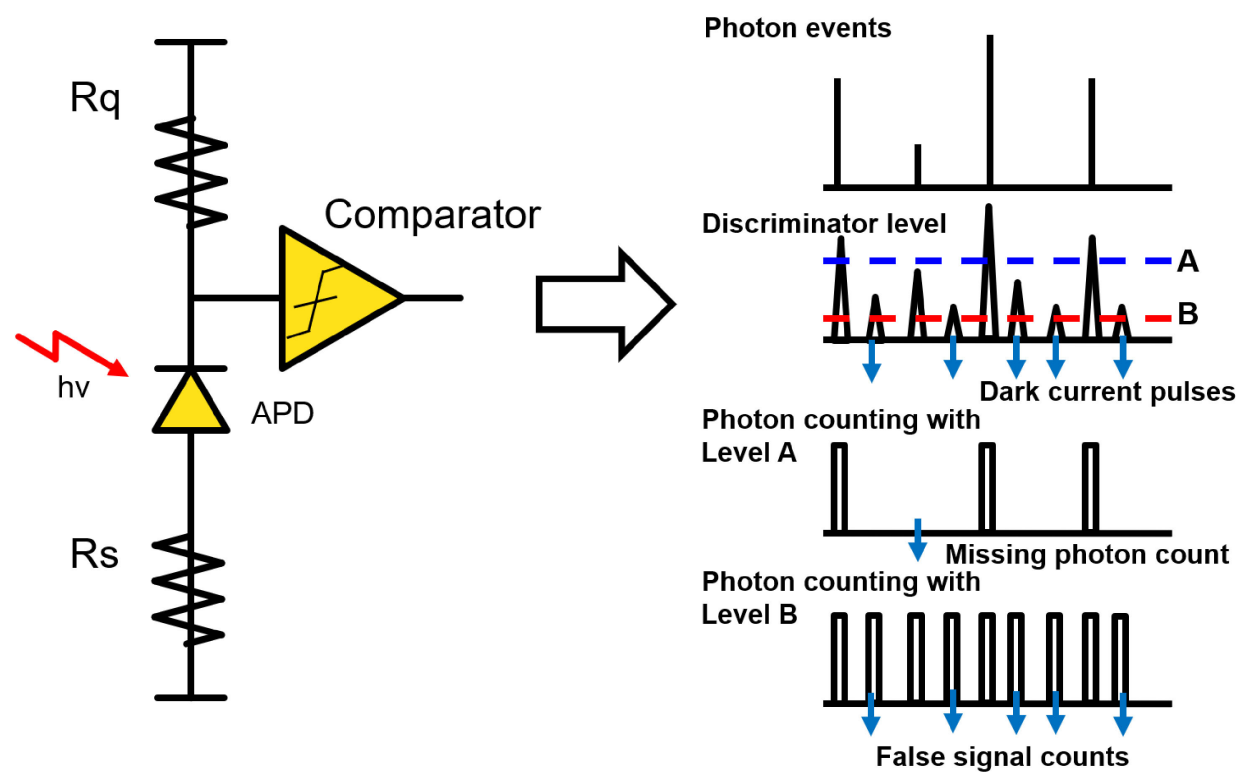

Fig. 1. Geiger mode resistive quenching circuit with a quenching resistor 
active reset topology can mitigate such problems but the quenching speed is still limited by the high resistance of $\mathrm{R}_{\mathrm{q}}[9,10]$. In addition, the quenching voltage level is linearly proportional to the photocurrent. When the photocurrent is low (i.e., weak signal), the discriminator voltage level of the comparator must be very small, which will increase the dark count probability (i.e., false signal count), as illustrated in Fig. 1.

In this work, a diode quenching element was proposed to improve the quenching performance.

\section{Experiments}

The resistive and diode quenching circuits used in SPICE simulation are shown in Fig. 2(a) and 2(b), respectively. The dashed boxes represent the APDs where the photocurrent input is provided by a pulsed current source in simulation [11]. The quenching resistor $\left(\mathrm{R}_{\mathrm{q}}\right)$ has a resistance of $100 \mathrm{k} \Omega$. It should be noted in Fig. 2(b) that the quenching diode is forward biased whereas the APD is reverse biased. The model parameters used in simulation for the APD are summarized in Table I. The APD is a SiC p-i-n APD with a breakdown voltage of $150 \mathrm{~V}$. It was assumed that the quenching diode was identical to the APD but had a reduced active area by one tenth. Therefore, the model parameters for the quenching diode were adjusted taking account of the reduced active area. The avalanche bias voltage was set to be $152 \mathrm{~V}$.

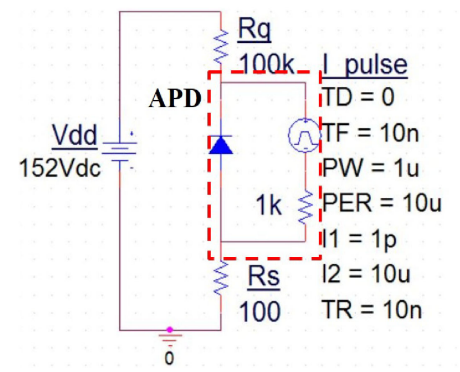

(a)

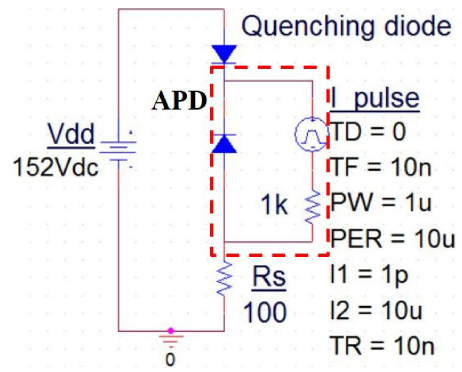

(b)

Fig. 2. Resistive and diode quenching circuits used in SPICE simulation

(a) Resistive quenching circuit

(b) Diode quenching circuit

The dependency of the quenching voltage levels of resistive and diode quenching circuits on the photocurrent are shown in Fig. 3(a) and 3(b). The dashed and solid lines represent the resistive and diode quenching circuits, respectively. While the quenching voltage level is linearly proportional to the photocurrent level in the resistive quenching circuit, it is independent of the photocurrent level in the diode quenching circuit. The quenching voltage level of the resistive quenching circuit is the product of the photocurrent and $R_{q}$, supposing that $R_{s}$ is negligible compared to $\mathrm{R}_{\mathrm{q}}$. On the other hand, the quenching voltage level of the diode quenching circuit is determined by the forward turn-on voltage of the quenching diode. Therefore, a constant quenching voltage level can be achieved by the diode quenching element. It is not a problem for the photon counting with an appropriate 
Table I. SPICE model parameters for PIN diode

\begin{tabular}{lc}
\hline Model Parameters & $10^{-10}$ \\
\hline Is (Saturation current) [A] & 10 \\
\hline $\mathrm{N}$ (Emission coefficient) & $1.98 \times 10^{-6}$ \\
\hline RS (Parasitic resistance) [S] & $1.98 \times 10^{-2}$ \\
\hline IKF (High-injection knee current) [A] & $1.81 \times 10^{-15}$ \\
\hline CJO (Zero-bias junction capacitance) [F] & $2.28 \times 10^{-1}$ \\
\hline M (Grading coefficient) & 3.18 \\
\hline VJ (Junction potential) [V] & $10^{-20}$ \\
\hline ISR (Recombination current) [A] & 6 \\
\hline NR (Emission coefficient for ISR) & 150 \\
\hline BV (Reverse breakdown voltage) [V] & $10^{-7}$ \\
\hline IBV (Current at BV) [A] & 0.5 \\
\hline FC (Forward-bias depletion capacitance coefficient) & $3.5 \times 10^{-12}$ \\
\hline TT (Transient time) [s] & 3 \\
\hline XTI (Is temperature exponent) & 3.25 \\
\hline EG (Bandgap energy) [eV] & \\
\hline
\end{tabular}

discriminator voltage level of the comparator. However, it should be noted that when the photocurrent becomes low, the discriminator voltage level must be very small, increasing the dark count probability.

The quenching transient characteristics of resistive and diode quenching circuits are compared in Fig. 3(c). The bias voltage across the APD falls down when the photocurrent is introduced. It is observed that the quenching times of the diode quenching circuit are significantly shorter than those of the resistive quenching circuit. The quenching and recovery times for the same quenching voltage level of $3 \mathrm{~V}$ are compared in Table II where the quenching and recovery times were defined when the voltage levels reached $90 \%$ of the final levels. The faster quenching speed is mainly attributed to the dynamic on-resistance characteristics of the forward biased quenching diode, which is much smaller than the $\mathrm{R}_{\mathrm{q}}$ of the resistive quenching circuit. One drawback of the diode quenching circuit is a relatively slower recovery time (rise time) after quenching. However, such a problem can be easily resolved by employing an active reset circuit that forces the APD bias voltage back to the avalanche bias voltage immediately [12].

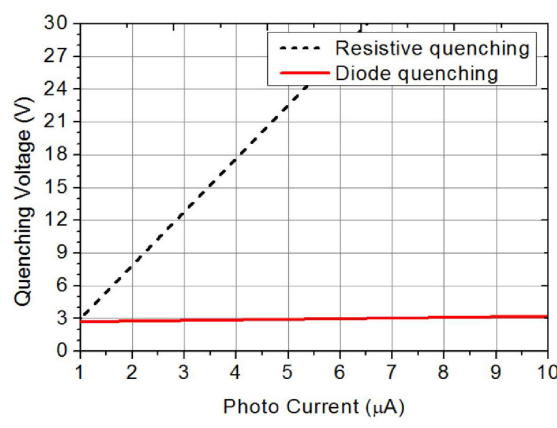

(a)

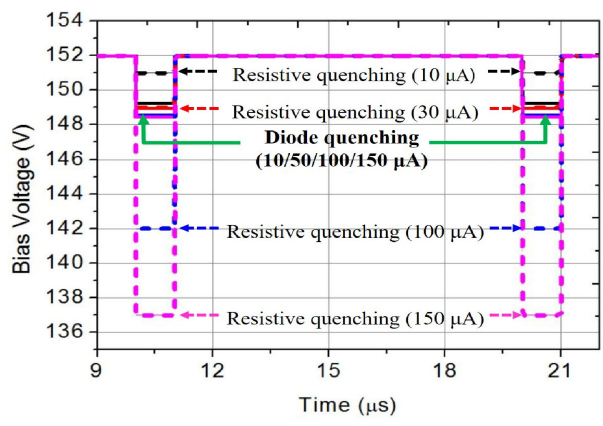

(b) 


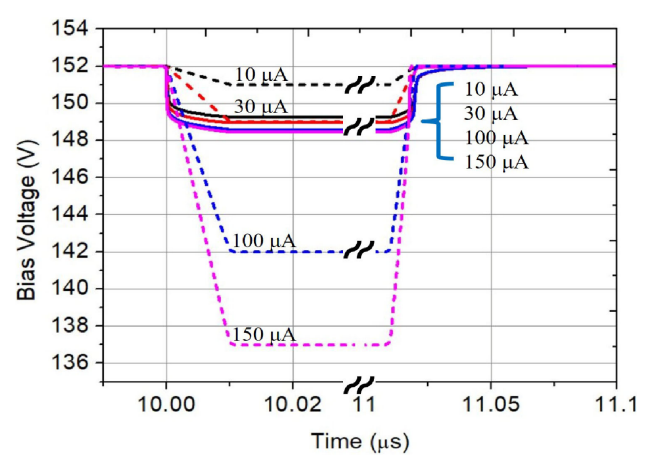

(c)

Fig. 3. Quenching characteristics of resistive and diode quenching circuits (Dashed lines: resistive quenching. Solid lines: diode quenching)

(a) Quenching voltage levels of resistive and diode quenching circuits as a function of photocurrent

(b) Comparison of quenching waveforms between resistive and diode quenching circuits simulated with different photocurrent levels

(c) Transient characteristics of resistive and diode quenching circuits with different photocurrent levels

Table II. Quenching and recovery times of resistive and diode quenching circuits for the quenching voltage of $3 \mathrm{~V}$

\begin{tabular}{lcc}
\hline Transient time & Resistive quenching & Diode quenching \\
\hline Quenching time (ns) & 9.46 & 1.06 \\
\hline Recovery time (ns) & 9.46 & 21 \\
\hline
\end{tabular}

\section{Conclusions}

A diode quenching circuit was proposed to provide a constant quenching voltage level. The quenching voltage level is independent of the photocurrent level due to the forward turn-on characteristics of the quenching diode, which enables stable quenching operation. In addition, faster quenching speed can be achieved by the low on-resistance under the forward turn-on condition of the quenching diode.

\section{Acknowledgement}

This work was supported by the Agency for Defense Development of Korea and Basic Science Research Program (No. 2015R1A6A1A03031833). 